\title{
Decubitus CT Myelography for CSF-Venous Fistulas: A Procedural Approach
}

\author{
(D) M.D. Mamlouk, (D) R.P. Ochi, (DP. Jun, and (DP.Y. Shen
}

\section{ABSTRACT}

SUMMARY: Decubitus CT myelography is a reported method to identify CSF-venous fistulas in patients with spontaneous intracranial hypotension. One of the main advantages of decubitus CT myelography in detecting CSF-venous fistulas is using gravity to dependently opacify the CSF-venous fistula, which can be missed on traditional myelographic techniques. Most of the CSF-venous fistulas in the literature have been identified in patients receiving general anesthesia and digital subtraction myelography, a technique that is not performed at all institutions. In this article, we discuss the decubitus CT myelography technique and how to implement it in daily practice.

ABBREVIATIONS: CTM = CT myelography; DSM = digital subtraction myelography; $\mathrm{SIH}=$ spontaneous intracranial hypotension

A spinal CSF-venous fistula is an abnormal connection that usually occurs between a meningeal diverticulum and a paraspinal vein that results in continuous shunting of CSF fluid and spontaneous intracranial hypotension (SIH). ${ }^{1}$ CSF-venous fistulas are 1 of the 3 types of CSF leaks, while CSF leaks from dural tears and ruptured meningeal diverticula are the remaining types. ${ }^{2}$ While the incidence of CSF-venous fistulas was originally thought to be rare, they may be present in a quarter of patients with SIH. ${ }^{3}$ In our experience and in the published literature, CSF-venous fistulas are under-recognized and are mostly discussed at only a few quaternary SIH academic centers in the United States.

Various diagnostics techniques have been reported to identify CSF-venous fistulas, which include conventional fluoroscopy and CT myelography (CTM), digital subtraction myelography (DSM), MR imaging myelography, and decubitus myelography in the aforementioned modalities. ${ }^{3-11}$ Of these techniques, decubitus CTM and DSM are the most frequently described in the literature to identify CSF-venous fistulas. The decubitus position permits contrast to flow into a CSF-venous fistula on the dependent side of the thecal sac via gravity. Because of this phenomenon,

Received June 3, 2020; accepted after revision August 12.

From the Department of Radiology (M.D.M., P.J., P.Y.S.), The Permanente Medical Group, Kaiser Permanente Medical Center, Santa Clara, Santa Clara, California; Department of Radiology and Biomedical Imaging (M.D.M.), University of California, San Francisco, San Francisco, California; and Department of Radiology (R.P.O.), The Permanente Medical Group, Kaiser Permanente Medical Center, Sacramento, Sacramento, California.

Please address correspondence to Mark D. Mamlouk, MD, Department of Radiology, The Permanente Medical Group, Kaiser Permanente Medical Center, Santa Clara, 700 Lawrence Expy, Santa Clara, CA 95051; e-mail:

mark.d.mamlouk@kp.org; @MarkMamloukMD

- Indicates open access to non-subscribers at www.ajnr.org

http://dx.doi.org/10.3174/ajnr.A6844
CSF-venous fistulas have been diagnosed on decubitus CTM and missed on prone or supine CTM. ${ }^{11}$ DSM has also used a similar advantage with the decubitus technique. In 1 study, a CSF-venous fistula was detected in 17 of 23 patients on decubitus DSM compared with 4 of 26 patients who underwent prone DSM. ${ }^{3}$ While both decubitus CTM and DSM can identify CSF-venous fistulas, there are no reported studies that have compared the sensitivities and specificities of detection.

There is a learning curve in some of these techniques, which may be challenging to perform and prevent radiologists from accurately diagnosing CSF-venous fistulas in daily practice. Kaiser Permanente Medical Center has explored many of the aforementioned myelographic techniques and found decubitus CTM to be a useful and relatively simple method to perform in most clinical practices and perhaps easier than DSM. There are many barriers to the DSM technique. First, familiarity with digital subtraction imaging is a requisite for DSM and may be challenging to implement if the operator does not have traditional interventional or neurointerventional training. Second, DSM is not performed at all institutions, and last, general anesthesia may be required. ${ }^{8}$ The purpose of this article is to discuss the procedural technique of decubitus CTM and when to perform it in a patient with SIH so that radiologists of various backgrounds can implement it in their practices.

\section{Patient Selection}

In patients with clinical suspicion and intracranial imaging findings consistent with SIH seen on a contrast-enhanced brain MR imaging, the referring provider at our institution is instructed to obtain a noncontrast total spine MR imaging. Our spine MR imaging technique consists of sagittal and axial T2-weighted 
imaging with a fat-suppression technique, which can detect extradural collections compatible with a CSF leak. ${ }^{12}$ Because the main reason for scanning these patients is to detect a CSF leak, we do not perform T1-weighted imaging or administer contrast because these sequences do not provide much additional value and unnecessarily increase the examination time. Contrast-enhanced sequences can better identify distended dural veins compared with the $\mathrm{T} 2$ sequence, but the $\mathrm{T} 2$ technique can differentiate the veins from a CSF leak: the former will be hypointense, and the latter, hyperintense. ${ }^{12}$ If MR imaging of the spine shows an extradural collection, the patient often has a fast CSF leak ${ }^{7}$ and we perform dynamic CTM with the patient in the prone Trendelenburg position using 1-2 $\mathrm{mL}$ of contrast, followed by targeted epidural blood patches. ${ }^{13}$ If MR imaging of the spine does not show an extradural collection, we perform decubitus CTM to exclude a CSF-venous fistula.

CSF-venous fistulas have been reported in a few patients with clinical symptoms of SIH but with normal brain MRI findings. ${ }^{8}$ We have occasionally performed decubitus CTM in patients with suspected SIH with normal brain MRI findings but have not found a CSF-venous fistula. To our knowledge, there are no specific myelographic recommendations in this patient cohort, and further studies are needed for guidance because cost, radiation, and the intervention itself are additional factors to consider.
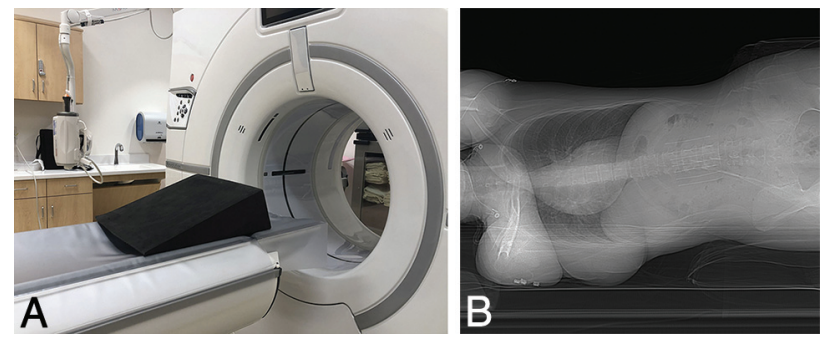

FIG 1. Patient positioning for decubitus CTM. A, A custom-made firm wedge with a $20^{\circ}$ angle is placed on the CT gantry, and the hips are placed at the apex of the wedge with the feet closest to the scanner bore. If no wedge is available, $1-2$ pillows can be placed instead. Pillows can also be used to support the legs. B, A lateral scout radiograph shows an adequate angle to facilitate contrast transit from the lumbar puncture to the remainder of the spine.

\section{Procedural Technique}

Decubitus Positioning. After review of the prior spine MR imaging and/or conventional myelograms if already performed, the operating physician decides whether to place the patient in the right or left decubitus position on the CT gantry table. This decision is usually predicated on which side has the greater number of enlarged or irregular-appearing meningeal diverticula on the prior imaging. While meningeal diverticula can occur normally in patients without $\mathrm{SIH}$, their existence may harbor a CSF-venous fistula in patients with $\mathrm{SIH}^{5}$ If the meningeal diverticula are approximately equal between the 2 sides or they are few in number, the right side is usually chosen because we have witnessed the right being more commonly implicated in our experience (82\%). Two additional studies ${ }^{6,14}$ have noted predominance on the right, while 1 study ${ }^{5}$ has reported CSF-venous fistulas more commonly occurring on the left.

We place the patient in the decubitus position with the use of a custom-made wedge, which is a firm sponge with a $20^{\circ}$ angle (Fig 1A). This wedge is chosen to facilitate flow of contrast from the lumbar puncture to the thoracic and cervical spine. If no wedge is available, 1-2 pillows can be placed under the patient's hips to achieve the same result. Pillows can also be placed under the patient's legs for comfort. Before proceeding with the lumbar puncture, the operating physician should observe that an adequate Trendelenburg angle $\left(10^{\circ}-20^{\circ}\right)$ is achieved. We also position the patient's hands above the head to prevent beamhardening while scanning. If the patient is unable to raise his or her hands for a long duration, then the hands can be placed by the side or chest and simply raised at the time of the total spine myelogram scanning.

Lumbar Puncture and Image Planning. We perform the lumbar puncture directly on the CT table as opposed to puncturing under fluoroscopy and then scanning with CT. The main reason for this direct technique is because we have observed that when the contrast mixes throughout the spinal canal and becomes more dilute, the CSF-venous fistula is more difficult to see. We believe this practice is one of the keys to successful imaging of CSF-venous fistulas. We also typically administer moderate sedation to our patients to make them comfortable during the procedure.

Initial scout images of the total spine are obtained in the frontal and lateral planes. We scrutinize the lateral scout image to ensure that there is an adequate $10^{\circ}-20^{\circ}$ angle before proceeding

Table: Demographics and imaging characteristics of patients with CSF-venous fistulas

\begin{tabular}{lclll}
\hline Patient & Age $(\mathbf{y r})$ & Sex & \multicolumn{1}{c}{ MR Imaging Findings } & Fistula Location \\
\hline 1 & 50 & M & Sag, dural enhancement, subdural collection & R T9-T10 \\
2 & 46 & F & Sag & R T10-T11 \\
3 & 90 & M & Sag, dural enhancement & R L1-L2 \\
4 & 61 & F & Dural enhancement & R T6-T7 \\
5 & 72 & F & Sag, dural enhancement, subdural collection & R T10-T11 \\
6 & 72 & F & Sag, dural enhancement, subdural collection & L T11-T12 \\
7 & 42 & F & Sag & R T8-T9 \\
8 & 39 & M & Sag & R T1-T2 \\
9 & 61 & F & Sag, dural enhancement & R L1-L2 \\
10 & 64 & M & Sag, dural enhancement, subdural collection & R T9-T10 \\
11 & 57 & M & Sag, dural enhancement, subdural collection & L T4-L5 \\
\hline
\end{tabular}

Note:- $R$ indicates right; $L$, left. 
with the lumbar puncture (Fig 1B). If the angle is suboptimal, then we reposition the patient. We subsequently obtain initial planning CT images of the lumbar spine and then typically puncture at the L2-L3 or L3-L4 levels with a 22-ga spinal needle.

Decubitus Myelographic Scanning. We administer $10 \mathrm{~mL}$ of preservative-free iohexol contrast (Omnipaque 300; GE Healthcare) for all decubitus CTMs. To ensure that the needle is in the subarachnoid space, we inject a test dose of approximately $0.5 \mathrm{~mL}$. Subsequently, we communicate with our CT technologists to prepare the next CT acquisition to encompass the total spine, using a $0.625-\mathrm{mm}$ section thickness and a diagnostic radiation dose with automatic exposure control. We have found that using a relatively larger section thicknesses can fail to capture subtle CSF-venous fistulas. We also typically acquire the CT images using standard or soft-tissue kernels, but a bone kernel can also be used, depending on user preference. After all the parameters are ready for scanning, we administer the remaining $9.5 \mathrm{~mL}$ of iohexol contrast, exit the CT scanning room, and scan immediately.

Most of our myelograms are obtained on either 16- or 64-section CT scanners, which contain an inherent adequate delay to ensure that the bolus has migrated throughout the spine. If one scans on faster CT machines, then one can consider a 15 -second delay to prevent scanning before the contrast bolus migration. We also specify our scanning phase to image from inferior to superior
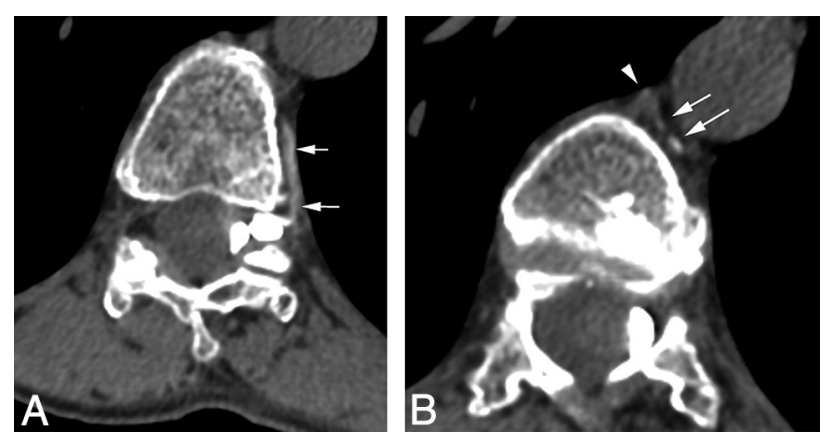

FIG 2. A CSF-venous fistula on decubitus CTM. A, Axial left-decubitus CTM shows a paravertebral vein (arrows) arising from a large left meningeal diverticulum at the $\mathrm{T} 11-\mathrm{T} 12$ level. $B$, On a more superior section, the vein (arrows) drains into the azygous vein (arrowhead).
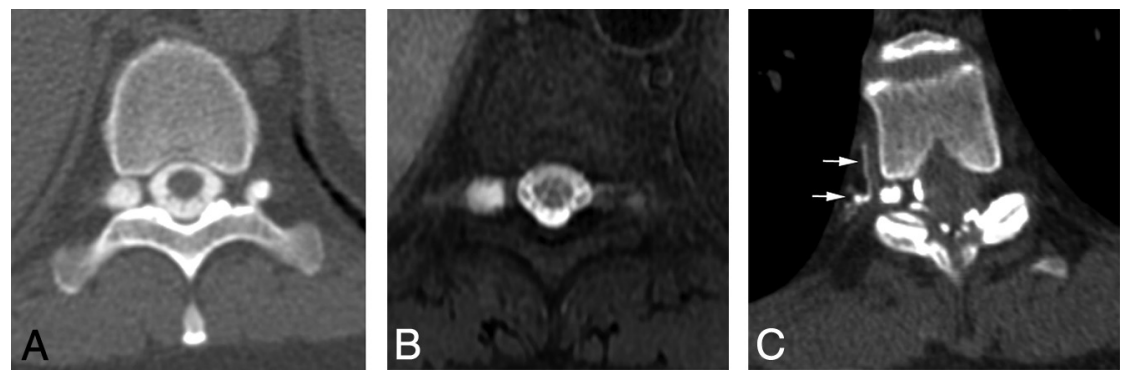

FIG 3. Value of decubitus CTM in diagnosing a CSF-venous fistula. A, An axial conventional CTM at the T10-T11 level shows bilateral meningeal diverticula without evidence of a CSF-venous fistula. $B$, An axial MR imaging myelogram with intrathecal gadolinium shows similar findings. $C$, A rightdecubitus CTM in the axial oblique plane shows a right-sided CSF-venous fistula with a paravertebral course (arrows). to coincide with the direction of the contrast bolus transit. We scrutinize the images directly on the CT technologist's workstation to ensure the following: 1) that the bolus has flowed to at least the mid-cervical spine, and 2) that we identified a CSF-venous fistula. If contrast did not flow adequately, then we sometimes manually position the patient in a more Trendelenburg fashion and then rescan immediately. We also occasionally rescan confirmed CSFvenous fistulas about 1-2 minutes after the initial acquisition if confirmation is needed.

If no CSF-venous fistula is identified, then we typically bring the patient back on the subsequent day and perform decubitus CTM of the contralateral side. There are 2 reasons for this practice: 1) There are dose constraints of administering more intrathecal contrast, ${ }^{15}$ and 2) when the patient is rotated to the contralateral side, the meningeal diverticula are less opacified because the contrast becomes more dilute after mixing throughout the spine. There are no published data comparing the practice of scanning the contralateral side in the same session versus in an additional session; however, at least 2 centers have reported the 2-day approach. ${ }^{3,9}$ Nonetheless, we are investigating whether performing the contralateral side scanning in the same session without additional contrast can still be successful, because it would be a more convenient practice for the patient and department. Last, we do not typically instruct the patient to perform any specific breathing during the myelogram acquisition, but in the future, we may scan with the inspiratory technique because this has been recently shown to increase conspicuity of CSF-venous fistulas in some cases. $^{16}$

\section{RESULTS}

During the past 16 months, we have performed decubitus CTMs on 22 patients and identified 11 patients with CSF-venous fistulas (Table). The mean age of patients with CSF-venous fistulas was 59 years (range, 39-90 years), and 6/11 were women. All patients with CSF-venous fistulas had at least 1 intracranial finding consistent with SIH, 10/11 had brain sag, 8/11 had dural enhancement, and 5/11 had subdural collections. Nine of 11 patients had right-sided CSF-venous fistulas, and 9/11 patients had thoracic CSF-venous fistulas, while the remaining 2 were at L1-L2. Of the $11 / 22$ patients who did not have CSF-venous fistulas on decubitus CTM, 5 of those patients had normal contrast-enhanced brain MRI findings and may have had an alternative diagnosis.

\section{Image Review and Reporting}

CSF-venous fistulas are nearly always seen in the thoracic spine, lower cervical spine, or high lumbar spine, ${ }^{5,6,17}$ and attention should be paid to these regions for fistula identification. In most cases, a CSF-venous fistula arises from a meningeal diverticulum, ${ }^{5}$ and the radiologist can identify a linear structure coursing from it that represents the abnormally connecting vein (Figs 2-6). CSF-venous fistula locations have been reported in 3 sites on CT: 

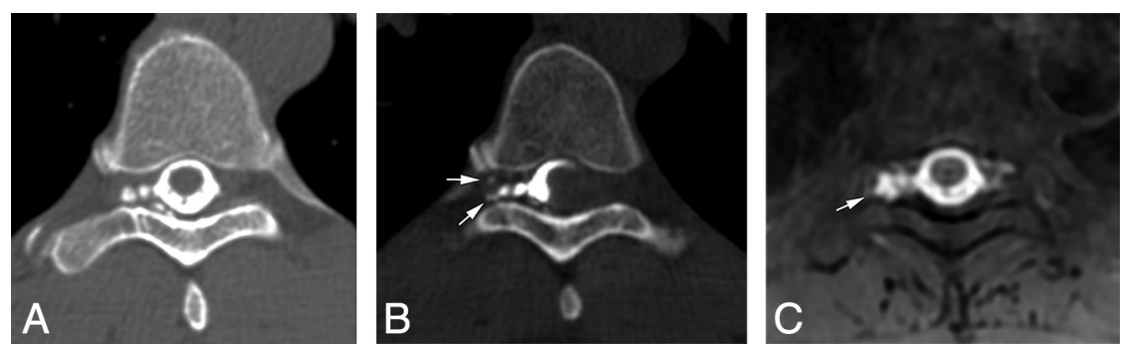

FIG 4. CSF-venous fistula on decubitus $C T$ and intrathecal MR imaging myelograms. $A$, An axial conventional CTM shows a large, irregular right meningeal diverticulum at the T8-T9 level but no abnormal vein. $B$, An axial right-decubitus CTM shows a CSF-venous fistula (arrows). C, An axial MR imaging myelogram with intrathecal gadolinium faintly shows the CSF-venous fistula (arrow), but it is not as apparent as the decubitus CTM.
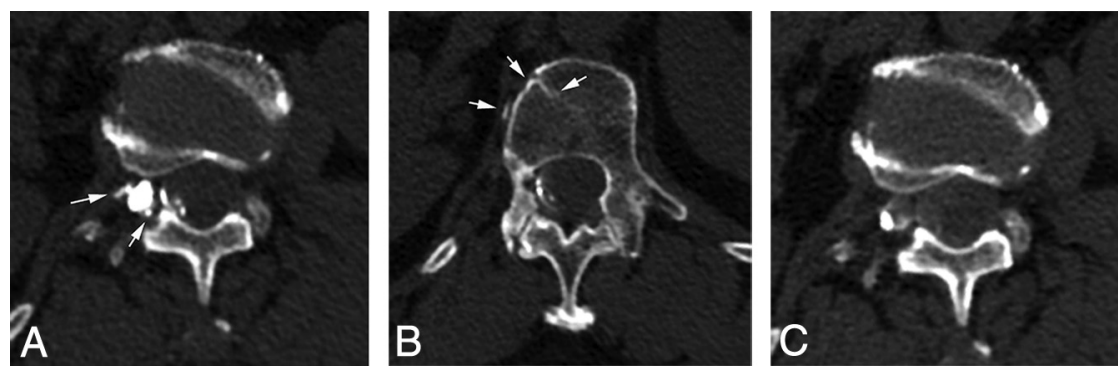

FIG 5. Importance of immediate scanning in decubitus CTM. A, An axial right-decubitus CTM scanned immediately after contrast injection shows abnormal veins (arrows) arising from a large right meningeal diverticulum at the L1-L2 level. B, On a more superior section, the vein (arrows) has a paravertebral course and terminates in the vertebral body. C, An axial right-decubitus CTM from a 3-minute delayed scan shows absence of the abnormal veins. If it were not for the initial scan, the CSF-venous fistula would have not been detected.
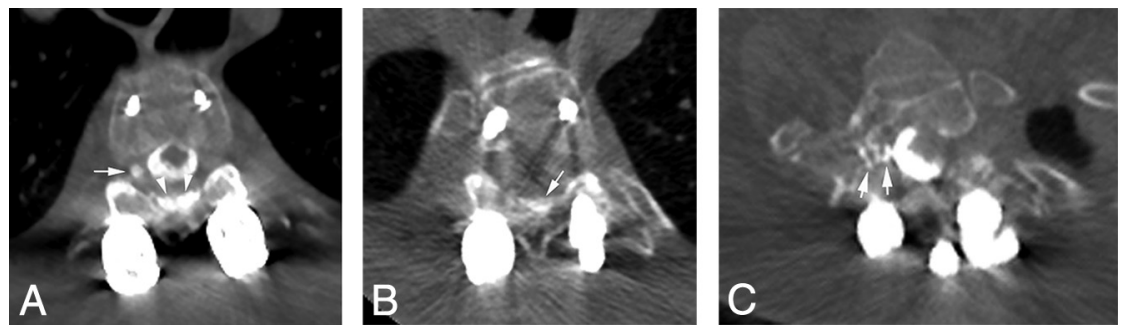

FIG 6. Value of precontrast imaging in a 39 year-old-man with a nearly 20 -year history of SIH. Ten years previously, the patient underwent surgical removal of a spinal venous malformation in the $\mathrm{Tl}$ posterior elements and dorsal epidural space, which was causing cord compression (not shown). $A$, An axial conventional CTM shows a round hyperdense focus in the right, lateral epidural space (arrow) and a broad hyperdense structure in the dorsal epidural space (arrowheads) at the T2-T3 level. This was suspicious for a CSF-venous fistula, but in the context of the surgical fusion, it was unclear whether the dorsal epidural hyperdensity was postsurgical. In the same session as the decubitus CTM, noncontrast imaging of the upper thoracic spine $(B)$ was performed, which confirmed that the dorsal epidural hyperdensity (arrow) was postsurgical. C, An axial oblique CT image from a right-decubitus CTM shows a large CSF-venous fistula (arrows) at the T1-T2 level, which is most likely associated with the prior venous malformation.

paravertebral, lateral (along the neural foramen and posterior elements), and central (within the internal vertebral epidural venous plexus). ${ }^{5}$ The paravertebral location was most commonly reported in 1 study, ${ }^{5}$ and this also coincides with our experience. The vein may be either single or cluster of veins and courses in multiple directions. Depending on the size of the vein and contrast timing, the abnormal vein can occasionally be seen draining into the azygous vein (Fig 2). We also scrutinize the myelogram for any additional fistulas or CSF leaks, though we have never seen either. Furthermore, if a CSF-venous fistula is identified, we do not image the contralateral side for additional fistulas because bilateral CSF-venous fistulas have not been reported, to our knowledge.

\section{CSF-Venous Fistulas on Additional Modalities}

In our experience, CSF-venous fistulas are more readily identifiable on decubitus CTM compared with conventional CTM. With the latter technique, the vein may often be absent (Figs 3, 4, and 6) or faintly shown. Furthermore, there may be false-positives with normal paravertebral veins, which can be a falselocalizing sign. ${ }^{18}$ Thus, we do not rely on conventional CTM for the assessment of CSF-venous fistulas. CSF-venous fistulas have been described in 3 patients with MR myelography after intrathecal gadolinium administration. ${ }^{10}$ Our institution occasionally uses this MR imaging technique for presumed slow CSF leaks that are occult on other myelographic techniques, and we have witnessed CSF-venous fistulas that were concordant on decubitus CTM and MR imaging myelography with intrathecal gadolinium (Fig 4); however, in our experience, MR imaging either usually fails to show the fistula (Fig 3) or shows multiple paravertebral veins that can make assessment difficult if used in isolation. Thus, we do not typically perform MR imaging myelography to diagnose CSFvenous fistulas.

DSM is an excellent technique to identify CSF-venous fistulas and is useful in capable hands, ${ }^{3,7,9,17}$ and we occasionally use this technique; however, in our experience, DSM requires a greater learning curve than decubitus CTM. In addition, if the radiologist is not wellversed in digital subtraction techniques or has training in traditional interventional or neurointerventional radiology, this may be more challenging to adopt in everyday practice. We believe that most radiologists of various academic and private practices can more readily perform decubitus CTM. DSM also sometimes necessitates the use of general anesthesia and may 
require 2 sessions of general anesthesia if the CSF-venous fistula is not identified in the first session. ${ }^{9}$ Thus, our institution relies on the decubitus CTM technique. To our knowledge, there are no reported studies that have compared the 2 techniques.

\section{Pitfalls and Tricks}

One of the major pitfalls of decubitus CTM is delayed imaging after intrathecal injection. We typically scan immediately after the injection of contrast to increase the likelihood of "catching" the CSF-venous fistula. The scanning parameters for the total spine CTM should be planned before the contrast injection so that the CSF-venous fistula is not missed. If more delayed scanning is performed, even a few minutes later, the fistula may be absent (Fig 5). This phenomenon suggests that performing decubitus CTM after fluoroscopy or DSM may have a lower yield, even if the patient's decubitus position is maintained between imaging modalities.

Calcification from the ligamentum flavum or high-density material from prior surgery can also mimic CSF-venous fistulas and leaks, and precontrast imaging in the area of interest may be performed for confirmation (Fig 6).

Insufficient contrast migration to the cervical spine can be a pitfall with decubitus CT myelography. We occasionally experience this if the Trendelenburg angle is not sufficient. In this event, we elevate the patient's trunk and legs to facilitate contrast to flow to the cervical spine and repeat scanning in the ROI immediately.

Early in our practice of decubitus CTM, we would occasionally inject intrathecal sterile saline if the spinal pressure was low to maximize visualization of the CSF-venous fistula before intrathecal contrast administration. Subsequently, we have realized that this practice did not improve identification of CSF-venous fistulas, and we have stopped it.

\section{CONCLUSIONS}

Decubitus CTM is a useful technique to identify CSF-venous fistulas in patients with SIH. The decubitus CTM technique has slight modifications compared with conventional myelography and can be used by radiologists of various practices to increase detection of CSF-venous fistulas.

\section{REFERENCES}

1. Schievink WI, Moser FG, Maya MM. CSF-venous fistula in spontaneous intracranial hypotension. Neurology 2014;83:472-73 CrossRef Medline

2. Schievink WI, Maya MM, Jean-Pierre S, et al. A classification system of spontaneous spinal CSF leaks. Neurology 2016;87:673-79 CrossRef Medline
3. Schievink WI, Maya MM, Moser FG, et al. Lateral decubitus digital subtraction myelography to identify spinal CSF-venous fistulas in spontaneous intracranial hypotension. J Neurosurg Spine 2019 Sept 13. [Epub ahead of print] CrossRef Medline

4. Kranz PG, Amrhein TJ, Schievink WI, et al. The "hyperdense paraspinal vein" sign: a marker of CSF-venous fistula. AJNR Am J Neuroradiol 2016;37:1379-81 CrossRef Medline

5. Kranz PG, Amrhein TJ, Gray L. CSF venous fistulas in spontaneous intracranial hypotension: imaging characteristics on dynamic and CT myelography. AJR Am J Roentgenol 2017;209:1360-66 CrossRef Medline

6. Clark MS, Diehn FE, Verdoorn JT, et al. Prevalence of hyperdense paraspinal vein sign in patients with spontaneous intracranial hypotension without dural CSF leak on standard CT myelography. Diagn Interv Radiol 2018;24:54-59 CrossRef Medline

7. Farb RI, Nicholson PJ, Peng PW, et al. Spontaneous intracranial hypotension: a systematic imaging approach for CSF leak localization and management based on MRI and digital subtraction myelography. AJNR Am J Neuroradiol 2019;40:745-53 CrossRef Medline

8. Schievink WI, Moser FG, Maya MM, et al. Digital subtraction myelography for the identification of spontaneous spinal CSF-venous fistulas. J Neurosurg Spine 2016;24:960-64 CrossRef Medline

9. Kim DK, Brinjikji W, Morris PP, et al. Lateral decubitus digital subtraction myelography: tips, tricks, and pitfalls. AJNR Am J Neuroradiol 2020;41:21-28 CrossRef Medline

10. Chazen JL, Robbins MS, Strauss SB, et al. MR myelography for the detection of CSF-venous fistulas. AJNR Am J Neuroradiol 2020;41:938-40 CrossRef Medline

11. Kranz PG, Gray L, Amrhein TJ. Decubitus CT myelography for detecting subtle CSF leaks in spontaneous intracranial hypotension. AJNR Am J Neuroradiol 2019;40:754-56 CrossRef Medline

12. Watanabe A, Horikoshi T, Uchida M, et al. Diagnostic value of spinal MR imaging in spontaneous intracranial hypotension syndrome. AJNR Am J Neuroradiol 2009;30:147-51 CrossRef Medline

13. Mamlouk MD, Shen PY, Jun P, et al. Developing a spinal CSF leak program in a multihospital network. Curr Probl Diagn Radiol 2020 April 9. [Epub ahead of print] CrossRef Medline

14. Duvall JR, Robertson CE, Cutsforth-Gregory JK, et al. Headache due to spontaneous spinal cerebrospinal fluid leak secondary to cerebrospinal fluid-venous fistula: case series. Cephalalgia 2019;39:1847-54 CrossRef Medline

15. Omnipaque (iohexol). https://www.gehealthcare.com/products/ contrast-media/omnipaque. Accessed May 29, 2020

16. Amrhein TJ, Gray L, Malinzak MD, et al. Respiratory phase affects the conspicuity of CSF-venous fistulas in spontaneous intracranial hypotension. AJNR Am J Neuroradiol 2020;41:1754-56 CrossRef Medline

17. Schievink WI, Maya MM, Moser FG. Digital subtraction myelography in the investigation of post-dural puncture headache in 27 patients: technical note. J Neurosurg Spine 2017;26:760-64 CrossRef Medline

18. Schievink WI, Maya MM, Moser FG. False localizing signs of spinal CSF-venous fistulas in spontaneous intracranial hypotension: report of 2 cases. J Neurosurg Spine 2019 July 5. [Epub ahead of print] CrossRef Medline 